\title{
Numerical simulation of shear band localization in geotechnical materials based on a nonlocal plasticity model
}

\author{
Shouxin $\mathrm{WU}^{1,2^{*}}$, Xingran $\mathrm{WANG}^{2}$ \\ 1. School of Civil Engineering, Southwest Jiaotong University, Chengdu 610031, China \\ 2. Trenchless Technology Center, Louisiana Tech University, Ruston, LA 71272, USA
}

\begin{abstract}
A new nonlocal plasticity model, which is based on the integral-type nonlocal model and the cubic representative volumetric element (RVE), is proposed to simulate shear band localization in geotechnical materials such as soils and rocks. An algorithm is developed to solve the resulting nonlinear system of equations. In this algorithm, the nonlocal averaging of plastic strain over the RVE is evaluated using $C^{0}$ elements instead of using $C^{1}$ elements to solve the second-order gradient of plastic strains. To obtain the average plastic strain, a set of special elements, called the nonlocal elements, are constructed to approximate the RVE. The updating of average stresses of the local element is based on the nonlocal plastic strain of the corresponding nonlocal elements. Numerical examples show that meshindependent results can be achieved using the proposed model and the algorithm, and the thickness of the shear band is insensitive to the mesh refinement.
\end{abstract}

Key words: nonlocal plasticity; shear band localization; finite element; gradient plasticity; nonlocal element

(C) 2011 JMT. All rights reserved.

\section{Introduction}

$\mathrm{S}$ hear band formation has been widely observed in the failure processes of geotechnical materials, such as concrete, rocks, and soils [1-4]. Numerical simulation of localization phenomena provides not only an approach to predict the onset, propagation, and final failure patterns of these materials, but also a means to examine and understand the failure mechanisms of these materials when they are losing the material stability [5-7].

It is widely recognized that the numerical modeling of the shear band localization based on classical continuum mechanics leads to pathological mesh-dependent solutions, which are caused by the loss of ellipticity of the governing differential equations [2,8-9]. From a mathematical point of view, the boundary value problem becomes ill-posed when the classical continuum models are employed to describe the mechanical responses of the materials with heterogenous properties. Generally speaking, these heterogenous materials experience inhomogenous deformation with high strain gradient occurring when loaded beyond the peak point [7-11].

Received Jul. 27, 2011; revision accepted Sep. 2, 2011

*Corresponding author. E-mail: swu2010@yahoo.com (S.X. WU)

(C) 2011 JMT. All rights reserved doi: 10.3969/j.issn.2095-087X.2011.03.007
A wide class of theories, which can be called nonlocality-based continuum theory, has been proposed to preserve the ellipticity of the governing equations and to regularize the mesh-dependency. Among the popular theories of this kind are Cosserat continuum theory [8,12], Toupin-Mindlin's micropolar theory [13-14], high-order gradient theory [15-17] and Eringen-Bažant's nonlocal theory [18-22].

Cosserat continuum theory treats material points as objects that have not only translational, but also rotational, degrees of freedom. This theory leads to additional strain quantities - independent relative rotation and micro-curvature, and additional stress quantitiesthe couple stresses, entering the constitutive description $[8,12]$. Cosserat continuum theories have been successfully used in the mesh-independent simulation of shear band localization. However, the rotational degrees of freedom are activated only under shear forces, which is the situation when the sliding of particle boundaries dominates the inelastic deformation (mode-II failure). If the decohesion, rather than frictional slip, dominates failure mode, the Cosserat theory is too weak to preserve the ellipticity of the governing differential equations. Toupin-Mindlin's micropolar theory is a generalized Cosserat theory, in which microscopic deformation gradient at the particle level and macroscopic deformation gradient at the structural level are both considered in the constitutive equations [13-14]. In Toupin- 
Mindlin's model, the first, second, and possibly higherorder gradients of strain tensors and conjugated stress tensors enter the constitutive equations, resulting in the so-called strain-gradient elasticity theory. This theory is further elaborated and extended to the plastic field, and gives rise to the development of gradient plasticity theory $[12,23]$. Moreover, the introduction of the second and higher-order gradient of internal state variables into the constitutive equations results in the well-known higher-order gradient theory [15-16]. Actually, higherorder gradient theory can be seen as a particular case of Toupin-Mindlin's micropolar theory. Although both theories can regularize the mesh-dependency, they impose higher-order continuity requirement on the interpolation function of internal state variables in the finite element context. For example, to evaluate the secondorder gradients of internal state variables, generally $C^{1}$ finite elements have to be used, while $C^{0}$ finite elements are sufficient for the standard discretization of the displacement field [24-25]. This mixed use of finite elements with different continuity properties incurs substantial increment of the number of degrees of freedom and the computational complexity.

Eringen-Bažant's nonlocal theory takes into account the behavior of microstructure of materials and longrange interactions between material particles by statistically averaging constitutive quantities [18-19,23,26]. The underlying assumption of the nonlocal theory is that the stress at a reference point in the body depends not only on strain history at that point, but also on strain histories at all other points of the body [23]. If the plastic strains are kept nonolocal and the other quantities are local, the nonlocal plasticity model results. The nonlocal model was first applied to the analysis of strain localization by Bažant and his co-workers [20-22]. In Bažant's integral-type nonlocal model, nonlocal counterpart of a field variable at a material point, e.g. nonlocal strain, is expressed as the weighted averaging of the local variable over a spatial neighborhood of that point [26].

Mathematically, the higher-order gradient model can be considered as the differential form of the integraltype nonlocal model [26]. If the local variable is substituted by its truncated Taylor series, the integral-type nonlocal model becomes the higher-order gradient models [26-27]. Therefore, the higher-order gradient models can be viewed as the approximate equivalents of the nonlocal models. This mathematical equivalence provides the possibility to combine the advantages of both the integral-type nonlocal model and the differentialtype nonlocal model, so that the difficulty of evaluating higher-order gradient term by $C^{1}$ elements in the finite element context can be circumvented.

Compared with other nonlocal models, the integraltype nonlocal model can address the material heteroge- neity phenomenologically in a statistically average sense. Through integral-type nonlocal models, a material representative volumetric element (RVE) having a length scale (the macroscale) that is sufficiently large compared to the microstructural length scale (the microscale), can be introduced in the constitutive equations. The material within the RVE can be accurately treated as being homogeneous.

The purpose of the present study is to propose a new nonlocal plasticity model based on the nonlocal theory and develop an effective algorithm to simulate shear band localization with an attempt to use only $C^{0}$ elements in the finite element solution. A cubic representative volumetric element (CRVE) is used to develop an integral-type nonlocal plasticity model and the internal length scale is associated with the characteristic length of the RVE. To avoid solving the second-order gradient of plastic multiplier using $C^{1}$ elements, the nonlocal plastic multiplier is evaluated as the averaging of the local plastic multiplier over a set of nonlocal elements. The stresses of the local element are updated based on the nonlocal plastic strains of the corresponding nonlocal elements. The moving elasto-plastic boundary technique is proposed to track the evolution of the plastic region. Two numerical examples of plane strain problems are carried out to illustrate the proposed model and the algorithm.

\section{Nonlocal plasticity model}

\subsection{Introduction to the nonlocal theory and CRVE}

Let $\boldsymbol{x}$ represent the position of a material point in a global reference system and $\xi$ the position of a material point in a local reference system with its origin located at $\boldsymbol{x}$. For a local field, $Y(\boldsymbol{x})$, defined in domain $\Omega$, its corresponding nonlocal counterpart, $\tilde{Y}(\boldsymbol{x})$, is defined as $[23,26]$

$$
\begin{aligned}
& \tilde{Y}(\boldsymbol{x})=\frac{1}{\tilde{V}_{\mathrm{RVE}}(\boldsymbol{x})} \int_{\Omega_{\mathrm{RVE}}(\boldsymbol{x})} w(\boldsymbol{x}, \boldsymbol{\xi}) Y(\boldsymbol{x}+\boldsymbol{\xi}) \mathrm{d} V, \\
& \forall: \boldsymbol{x} \in \Omega ; \boldsymbol{\xi} \in \Omega_{\mathrm{RVE}}(\boldsymbol{x}),
\end{aligned}
$$

in which

$$
\tilde{V}_{\mathrm{RVE}}(\boldsymbol{x})=\int_{\Omega_{\mathrm{RVE}}(\boldsymbol{x})} w(\boldsymbol{x}, \boldsymbol{\xi}) \mathrm{d} V,
$$

where $\Omega_{\mathrm{RVE}}(\boldsymbol{x})$ represents the domain occupied by a $\mathrm{RVE}$ at the reference material point $\boldsymbol{x}, w(\boldsymbol{x}, \boldsymbol{\xi})$ is a nonlocal weighting function over $\Omega_{\mathrm{RVE}}(\boldsymbol{x})$. The sym- 
bol " " over a variable denotes the nonlocal counterpart of the local variable.

The nonlocal weighting function $w(\boldsymbol{x}, \boldsymbol{\xi})$ is a nonnegative function and monotonically decreases with increased $\|\boldsymbol{\xi}\|$. At $\|\boldsymbol{\xi}\|=0, w(\boldsymbol{x}, \boldsymbol{\xi})$ acquires its maximum and when $\|\boldsymbol{\xi}\| \rightarrow \infty, w(\boldsymbol{x}, \boldsymbol{\xi}) \rightarrow 0$.

Consider a cubic representative volumetric element (CRVE) with a side length of $l_{\mathrm{c}}$ (Fig. 1). Suppose that the center of this CRVE is located at $\boldsymbol{x}^{o}=\left(x_{1}^{o}, x_{2}^{o}, x_{3}^{o}\right)$ in the global Cartesian coordinate system and serves as the origin of a local Cartesian coordinate system $\boldsymbol{\xi}=\left(\xi_{1}, \xi_{2}, \xi_{3}\right)$. If the field $Y(\boldsymbol{x})$ is sufficiently smooth within this CRVE, it can be expanded as the following Taylor series:

$$
\begin{gathered}
Y\left(\boldsymbol{x}^{o}+\boldsymbol{\xi}\right)=Y\left(\boldsymbol{x}^{o}\right)+\left(\left.\frac{\partial Y(\boldsymbol{x})}{\partial x_{i}}\right|_{\boldsymbol{x}=\boldsymbol{x}^{o}}\right) \xi_{i}+ \\
\frac{1}{2}\left(\left.\frac{\partial^{2} Y(\boldsymbol{x})}{\partial x_{i} \partial x_{j}}\right|_{\boldsymbol{x}=\boldsymbol{x}^{o}}\right) \xi_{i} \xi_{j}+O\left(\xi_{i} \xi_{j} \xi_{k}\right),
\end{gathered}
$$

where the Einstein's summation convention applies to the dummy indices.

Generally, for every material point at $x \in \Omega$, there is a CRVE centered at $\boldsymbol{x}$. If $Y(\boldsymbol{x})$ is smooth enough for all $\boldsymbol{x} \in \Omega$, then Eq. (3) is valid for $\boldsymbol{x} \in \Omega$ and $\boldsymbol{\xi} \in \Omega_{\mathrm{RVE}}(\boldsymbol{x})$. Replacing $\boldsymbol{x}^{o}$ with $\boldsymbol{x}$ and substituting Eq. (3) and $w(\boldsymbol{x}, \boldsymbol{\xi})=1$ (uniform distribution function) into Eqs. (1) and (2), with the fourth and higher-order terms neglected, the following differential equation results [27-28]

$$
\tilde{Y}(\boldsymbol{x})=Y(\boldsymbol{x})+\frac{l_{\mathrm{c}}^{2}}{24} \nabla^{2} Y(\boldsymbol{x}),
$$

where $l_{\mathrm{c}}$ is defined as the side length of a CRVE and often called the characteristic length of the CRVE. Eq. (4) is an approximate expression of the integral-type nonlocal model, as given by Eq. (1).

In the literature of nonlocal or gradient theory [1516,28], an internal length scale $c$ is usually defined through

$$
\tilde{Y}(\boldsymbol{x})=Y(\boldsymbol{x})+c^{2} \nabla^{2} Y(\boldsymbol{x}) .
$$

Comparing Eq. (4) with Eq. (5), one obtains

$$
c=\frac{l_{\mathrm{c}}}{\sqrt{24}} .
$$

It is noted that through Eq. (4) and CRVE, a link between the nonlocal integral-type model and a differential equation is established, and the nonlocal effect can be introduced to some degree by adding a second-order gradient of the local field into the original local field. In a later section we will show that it is just because of this link that makes it possible to use $C^{0}$ elements to solve the nonlocal field.

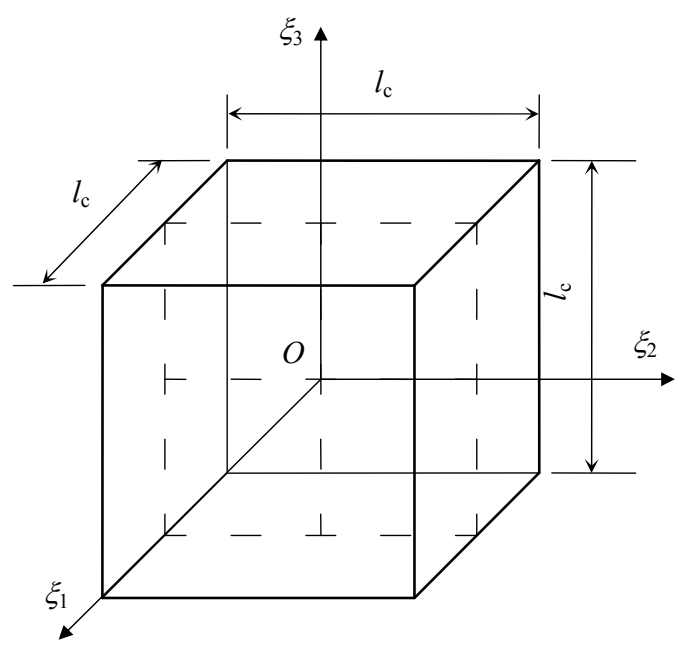

Fig. 1 The cubic representative volumetric element (CRVE)

\subsection{The nonlocal plasticity}

In the nonlocal plasticity theory, the yield function in the stress space at a material point generally depends on the local stress state and the nonlocal internal state variables at that material point $[23,26]$ :

$$
\begin{aligned}
& f(\sigma, \tilde{\eta}):=\mathscr{F}(\boldsymbol{\sigma})-\sigma_{\mathrm{Y}}(\tilde{\eta})=0, \\
& \sigma_{\mathrm{Y}}(\tilde{\eta})=\sigma_{\mathrm{Y} 0}+\chi(\tilde{\eta}),
\end{aligned}
$$

where $\tilde{\eta}$ and $\eta$ are related by Eq. (4), which can be rewritten as:

$$
\tilde{\eta}(\boldsymbol{x})=\eta(\boldsymbol{x})+\frac{l_{\mathrm{c}}^{2}}{24} \nabla^{2} \eta(\boldsymbol{x}),
$$

where $\sigma$ is a Cauchy stress tensor, $\eta$ denote an local internal variable, which is a nonnegative scalar, and $\tilde{\eta}$ is its nonlocal counterpart; $\mathscr{F}(\sigma)$ represents an equivalent stress; $\sigma_{\mathrm{Y}}(\tilde{\eta})$ is the yield stress, and $\chi(\tilde{\eta})$ is the evolution function, which is defined as

$$
\left.\begin{array}{l}
\chi(\tilde{\eta})>0: \text { Strain hardening plasticity } \\
\chi(\tilde{\eta})=0: \text { Perfect plasticity } \\
\chi(\tilde{\eta})<0: \text { Strain Softening plasticity }
\end{array}\right\} \forall: \tilde{\eta}>0 .
$$




\section{Finite element formulation of the two coupled fields}

\subsection{Governing differential equations}

Suppose that at a given instant of time $t$, a domain $\Omega$ is in a state of static equilibrium and the state of stress $\sigma$ and its loading history is known throughout the domain. The external force increment $\mathrm{d} \hat{\boldsymbol{F}}$ is prescribed on $\partial_{g} \Omega$ and the displacement increment $\mathrm{d} \hat{\boldsymbol{u}}$ is prescribed on $\partial_{u} \Omega$, where $\partial_{g}$ and $\partial_{u}$ denote the boundary associated with the given forces and displacements (Fig. 2). According to the flow theory of plasticity, the boundary value problem of incremental elastoplasticity is to find the incremental displacement field $\mathrm{d} \boldsymbol{u}$ that satisfies the equation of equilibrium, in $\Omega$ [29-30],

$\operatorname{div} \mathrm{d} \boldsymbol{\sigma}+\mathrm{d} \boldsymbol{b}=0$

with the boundary conditions:

$$
\begin{aligned}
& \mathrm{d} \boldsymbol{\sigma} \cdot \boldsymbol{n}=\mathrm{d} \hat{\boldsymbol{F}} \text { on } \partial_{g} \Omega, \\
& \mathrm{d} \boldsymbol{u}=\mathrm{d} \hat{\boldsymbol{u}} \text { on } \partial_{u} \Omega,
\end{aligned}
$$

where $\mathrm{d} \boldsymbol{b}$ denotes the body force increment; $\boldsymbol{n}$ is the unit outward normal vector to $\partial_{g} \Omega$.

The strain-displacement relation and the constitutive relation are given by

$$
\begin{aligned}
& \mathrm{d} \boldsymbol{\varepsilon}=\frac{1}{2}\left(\nabla \mathrm{d} \boldsymbol{u}+(\nabla \mathrm{d} \boldsymbol{u})^{\mathrm{T}}\right), \\
& \mathrm{d} \boldsymbol{\sigma}=\boldsymbol{C}^{\mathrm{e}}:\left(\mathrm{d} \boldsymbol{\varepsilon}-\mathrm{d} \boldsymbol{\varepsilon}^{\mathrm{p}}\right),
\end{aligned}
$$

where $\partial_{g} \Omega \bigcup \partial_{u} \Omega=\partial \Omega, \partial_{g} \Omega \bigcap \partial_{u} \Omega=\emptyset$. The domain $\Omega$ can be divided into elastoplastic domain $\Omega_{\text {ep }}$ and elastic domain $\Omega_{\mathrm{e}}$, i.e. $\Omega=\Omega_{\mathrm{ep}} \cup \Omega_{\mathrm{e}}$. In elastic domain $\Omega_{\mathrm{e}}$, we have $\mathrm{d} \varepsilon^{\mathrm{p}}=0$. In elastoplastic domain $\Omega_{\text {ep }}$, when the material experiences unloading, we have $\mathrm{d} \varepsilon^{\mathrm{p}}=0$. If it experiences plastic loading, we have $\mathrm{d} \varepsilon^{\mathrm{p}}>0$. The plastic loading is assumed to obey the associative normal flow rule through

$$
\mathrm{d} \varepsilon^{\mathrm{p}}=\mathrm{d} \lambda \frac{\partial f}{\partial \boldsymbol{\sigma}} .
$$

The plastic loading/unloading condition obeys the following Kuhn-Tucker complementarity condition [29]

$$
\mathrm{d} \lambda \geq 0, f(\sigma, \tilde{\eta}) \leq 0, \text { and } \mathrm{d} \lambda f(\sigma, \tilde{\eta})=0,
$$

and satisfies the consistency requirement

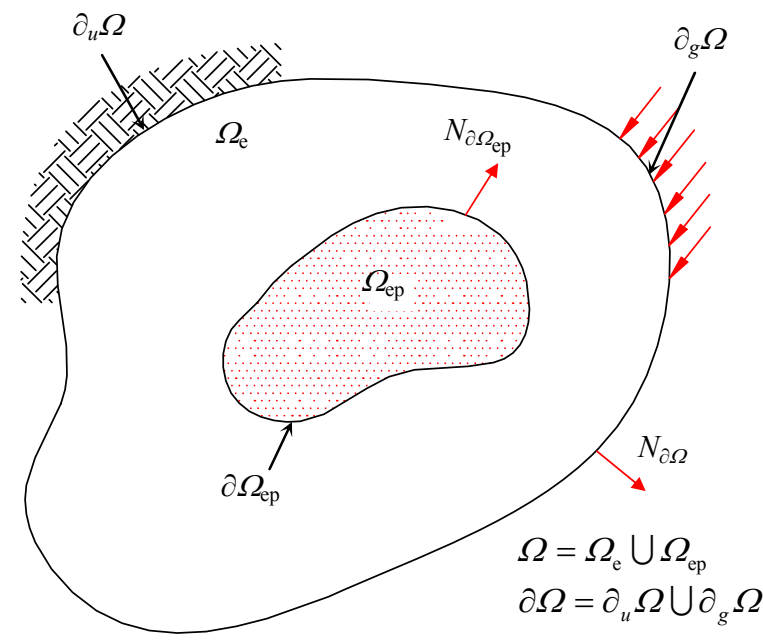

Fig. 2 Domain of two coupled elasto-plastic boundary-value problem

$\mathrm{d} \lambda \mathrm{d} f(\sigma, \tilde{\eta})=0$.

During plastic loading, $\mathrm{d} \lambda \geq 0$, Eq. (17) and Eq. (18) turn out to be

$$
\begin{gathered}
f(\sigma, \tilde{\eta})=0 \text { in } \Omega_{\text {ep }}, \\
\mathrm{d} f(\sigma, \tilde{\eta})=0 \text { in } \Omega_{\text {ep }} .
\end{gathered}
$$

By chain rule and nonlocal relation (5), Eqs. (7) and (9), consistency equation (20) can be rewritten as [2425,31-32]

$$
\frac{\partial f}{\partial \boldsymbol{\sigma}}: \mathrm{d} \boldsymbol{\sigma}-\frac{\partial \sigma_{\mathrm{Y}}(\tilde{\eta})}{\partial \tilde{\eta}}\left(\mathrm{d} \eta+c^{2} \nabla^{2} \mathrm{~d} \eta\right)=0
$$

If we define the plastic modulus as $E_{\mathrm{p}}=\partial \sigma_{\mathrm{Y}}(\tilde{\eta}) / \partial \tilde{\eta}=\partial \sigma_{\mathrm{Y}}(\eta) / \partial \eta$ and let $\boldsymbol{a}=\partial f / \partial \boldsymbol{\sigma}$ and $k=E_{\mathrm{p}} c^{2}$, we obtain

$$
\boldsymbol{a}: \mathrm{d} \boldsymbol{\sigma}-E_{\mathrm{p}} \mathrm{d} \eta-k \nabla^{2} \mathrm{~d} \eta=0
$$

which is a second-order differential equation in internal variable $\eta$ and only valid under the following conditions: $\boldsymbol{x} \in \Omega_{\mathrm{ep}}$ and $\eta>0$ (i.e. plastic loading).

To solve Eq. (22), the boundary condition related to $\eta(\boldsymbol{x})$ has to be prescribed on $\partial \Omega_{\mathrm{ep}}$. According to [24] and [33], for modeling strain localization, Dirichlet boundary conditions should be applied on $\partial \Omega_{\mathrm{ep}}$, that is

$$
\mathrm{d} \eta=0 \text { on } \partial \Omega_{\mathrm{ep}} .
$$

The boundary value problem of the field $\mathrm{d} \eta(x)$ is to find the internal variable $\eta(x)$ in domain $\Omega_{\text {ep }}$ that sat- 
isfies Eq. (22) and the boundary condition (23). The internal variable $\eta$ can be taken to be the equivalent plastic strain, $\varepsilon_{\text {eq }}^{\mathrm{p}}$,

$$
\mathrm{d} \eta=\mathrm{d} \varepsilon_{\mathrm{eq}}^{\mathrm{p}}
$$

where $\mathrm{d} \varepsilon_{\mathrm{eq}}^{\mathrm{p}}=\sqrt{\frac{2}{3} \mathrm{~d} \varepsilon_{i j}^{\mathrm{p}} \mathrm{d} \varepsilon_{i j}^{\mathrm{p}}}$. Under the associative normal flow rule and isotropic strain hardening/softening, for many yield functions the relation between $\mathrm{d} \eta$ and $\mathrm{d} \lambda$ has the linear form (see [24] and [31]): $\mathrm{d} \eta=\gamma \mathrm{d} \lambda$. For the von Mises yield function, one can derive $\gamma=1$ [24]. Thus Eq. (22) can be rewritten as a second-order differential equation with respect to incremental plastic multiplier $\mathrm{d} \lambda$ :

$$
\boldsymbol{a}: \mathrm{d} \boldsymbol{\sigma}-E_{\mathrm{p}} \mathrm{d} \lambda-k \nabla^{2} \mathrm{~d} \lambda=0
$$

From Eq. (25), it can be seen that two fields, the displacement field $\boldsymbol{u}$ and the plastic multiplier field $\lambda$ (or the internal variable field $\eta$ ), are coupled with each other through flow rule (16).

\subsection{Variational formulation of two coupled field equations}

To solve the two coupled fields numerically using finite element approximation, variational formulae of the field equations have to be derived. Following Mühlhaus and Aifantis [17] and de Borst and Mühlhaus [24], we can obtain the weak forms of the two coupled differential field equations (11) and (25) as follows:

$$
\left.\begin{array}{c}
\int_{\Omega} \delta \mathrm{d} \boldsymbol{\varepsilon}: \boldsymbol{C}:(\mathrm{d} \boldsymbol{\varepsilon}-\mathrm{d} \lambda \boldsymbol{a}) \mathrm{d} V=\int_{\partial_{\mathrm{g}} \Omega} \mathrm{d} \hat{\boldsymbol{F}} \cdot \delta \mathrm{d} \boldsymbol{u} \mathrm{d} S \\
\mathrm{~d} \boldsymbol{u}=\mathrm{d} \hat{\boldsymbol{u}} \quad \\
\text { on } \partial_{u} \Omega
\end{array}\right\} \forall: \boldsymbol{x} \in \Omega,
$$

The variational statement of these two coupled field equations should be defined as: to find $\mathrm{d} \boldsymbol{u}$ and $\mathrm{d} \lambda$ such that Eq. (26a) and Eq. (26b) are satisfied simultaneously.

\subsection{Galerkin's Formulation}

From this point on we will use \lfloor\rfloor to denote a row matrix and \{\} to denote a column matrix, and [ ] to denote the other matrices that are neither row matrices nor column matrices.

Let us discretize the domain $\Omega$ into element domain $\Omega^{e}, 1 \leq e \leq n_{\mathrm{el}}$, where $n_{\mathrm{el}}$ is the total number of elements. The displacement field $\mathrm{d} \boldsymbol{u}$ within $\Omega^{e}$ can be approximated by

$$
\{\mathrm{d} \boldsymbol{u}\}=[\boldsymbol{N}]\left\{\mathrm{d} \boldsymbol{u}_{\mathrm{h}}^{\mathrm{e}}\right\},
$$

where $[\boldsymbol{N}]$ is the shape function for displacement and $\left\{\mathrm{d} \boldsymbol{u}_{\mathrm{h}}^{\mathrm{e}}\right\}$ represents the displacements at element nodal points. It should be noted that the subscript " $h$ " means the discretized quantities, while the superscript "e" implies that the quantities are for a finite element. The strain-displacement relations can be expressed in the matrix form:

$$
\{\boldsymbol{\varepsilon}\}=[\boldsymbol{B}]\left\{\mathrm{d} \boldsymbol{u}_{\mathrm{h}}^{\mathrm{e}}\right\}
$$

For the same element domain $\Omega^{e}$, if $\Omega^{e} \subseteq \Omega_{\text {ep }}$ and $\mathrm{d} \lambda>0$, the plastic multiplier field $\mathrm{d} \lambda$ can be approximated by

$$
\{\mathrm{d} \lambda\}=\lfloor\boldsymbol{\Phi}\rfloor\left\{\mathrm{d} \lambda^{\mathrm{e}}\right\}
$$

where $\lfloor\boldsymbol{\Phi}\rfloor$ is the shape function of $\{\mathrm{d} \lambda\}$.

The gradient of $\{d \lambda\}$ is written as

$$
\nabla\{\mathrm{d} \lambda\}=[\nabla \boldsymbol{\Phi}]\left\{\mathrm{d} \lambda^{\mathrm{e}}\right\}=[\boldsymbol{\Psi}]\left\{\mathrm{d} \lambda^{\mathrm{e}}\right\}
$$

where

$$
[\boldsymbol{\Psi}]=[\nabla \boldsymbol{\Phi}] .
$$

Substituting Eqs. (27) to (31) into Eqs. (26a) and (26b), we obtain the following matrix equation (see [24,31] for details):

$$
\left[\begin{array}{cc}
\boldsymbol{K}_{u u}^{\mathrm{e}} & \boldsymbol{K}_{u \lambda}^{\mathrm{e}} \\
\left(\boldsymbol{K}_{u \lambda}^{\mathrm{e}}\right)^{\mathrm{T}} & \boldsymbol{K}_{\lambda \lambda}
\end{array}\right]\left\{\begin{array}{l}
\mathrm{d} \boldsymbol{u}_{\mathrm{h}}^{\mathrm{e}} \\
\mathrm{d} \lambda_{\mathrm{h}}^{\mathrm{e}}
\end{array}\right\}=\left\{\begin{array}{c}
\mathrm{d} \hat{\boldsymbol{F}}_{\mathrm{h}}^{\mathrm{e}} \\
0
\end{array}\right\},
$$

where 


$$
\begin{aligned}
& {\left[\boldsymbol{K}_{u u}^{\mathrm{e}}\right]=\int_{\Omega^{e}}[\boldsymbol{B}]^{\mathrm{T}}[\boldsymbol{C}][\boldsymbol{B}] \mathrm{d} V,} \\
& {\left[\boldsymbol{K}_{u \lambda}^{\mathrm{e}}\right]=-\int_{\Omega_{\mathrm{ep}}^{\mathrm{e}}}[\boldsymbol{B}]^{\mathrm{T}}[\boldsymbol{C}]\{\boldsymbol{a}\}\lfloor\boldsymbol{\Phi}\rfloor \mathrm{d} V,} \\
& {\left[\boldsymbol{K}_{\lambda \lambda}^{\mathrm{e}}\right]=\int_{\Omega_{\mathrm{ep}}^{e}}\left(\lfloor\boldsymbol{\Phi}\rfloor^{\mathrm{T}}\{\boldsymbol{a}\}^{\mathrm{T}}[C]\{\boldsymbol{a}\}\lfloor\boldsymbol{\Phi}\rfloor+\right.} \\
& \left.E_{\mathrm{p}}\lfloor\boldsymbol{\Phi}\rfloor^{\mathrm{T}}\lfloor\boldsymbol{\Phi}\rfloor-k[\boldsymbol{\Psi}]^{\mathrm{T}}[\boldsymbol{\Psi}]\right) \mathrm{d} V, \\
& \left\{\mathrm{~d} \hat{\boldsymbol{F}}_{\mathrm{h}}^{\mathrm{e}}\right\}=\int_{\partial_{g} \Omega^{e}}[\boldsymbol{N}]^{\mathrm{T}}\{\mathrm{d} \hat{\boldsymbol{F}}\} \mathrm{d} S .
\end{aligned}
$$

Notice that the element stiffness matrix is symmetric when the associative flow rule is applied. This makes it possible to take advantage of the existing symmetric equation solvers to save program coding work. Also, we notice that $C^{0}$ continuity is enough to find the solution of $\{\mathrm{d} \boldsymbol{u}\}$ and $\{\mathrm{d} \lambda\}$ in the finite element context.

\section{Solution strategy of the nonlinear system of equations}

\subsection{General methodology}

Matrix equation (32) comes from the weak forms of incremental equilibrium equations (12) and the consistency equation (25). And it is actually the linearized form of a nonlinear system of operator equations $[29,34]$. Strictly, the solutions of the two coupled fields, $\boldsymbol{u}$ and $\lambda$, must satisfy the following equilibrium equation (for convenience, we take body force $\boldsymbol{b}=0$ ):

$\operatorname{div} \boldsymbol{\sigma}=0$, in $\Omega$

with the boundary conditions $\boldsymbol{\sigma} \cdot \boldsymbol{n}=\hat{\boldsymbol{F}}$ on $\partial_{g} \Omega$ and $\boldsymbol{u}=\hat{\boldsymbol{u}}$ on $\partial_{u} \Omega$ and yield condition:

$$
f(\sigma, \tilde{\eta})=0 \text {, in } \Omega_{\mathrm{ep}} .
$$

Following the same method as the derivation of the weak form of the incremental equilibrium equation, we obtain the following matrix form of the equilibrium equation (37) [29,35]:

$$
\int_{\Omega^{e}}[\boldsymbol{B}]^{\mathrm{T}}[\boldsymbol{\sigma}] \mathrm{d} V-\int_{\partial_{g} \Omega^{e}}[\boldsymbol{N}]^{\mathrm{T}}\{\hat{\boldsymbol{F}}\} \mathrm{d} S=0 .
$$

During the $(k+1)$ th iteration, we require

$$
\int_{\Omega^{e}}[\boldsymbol{B}]^{\mathrm{T}}[\boldsymbol{\sigma}]_{k+1} \mathrm{~d} V-\int_{\partial_{g} \Omega^{e}}[\boldsymbol{N}]^{\mathrm{T}}\{\hat{\boldsymbol{F}}\}_{k+1} \mathrm{~d} S=0 .
$$

If $[\boldsymbol{\sigma}]$ and $\{\hat{\boldsymbol{F}}\}$ are linearly additive, i.e.,

$$
[\boldsymbol{\sigma}]_{k+1}=[\boldsymbol{\sigma}]_{k}+[\Delta \boldsymbol{\sigma}]
$$

$$
\{\hat{\boldsymbol{F}}\}_{k+1}=\{\hat{\boldsymbol{F}}\}_{k}+\{\Delta \hat{\boldsymbol{F}}\} .
$$

Equation (40) can be rewritten as

$$
\begin{gathered}
\int_{\Omega^{e}}[\boldsymbol{B}]^{\mathrm{T}}[\Delta \boldsymbol{\sigma}] \mathrm{d} V=\int_{\partial_{g} \Omega^{e}}[\boldsymbol{N}]^{\mathrm{T}}\{\Delta \hat{\boldsymbol{F}}\} \mathrm{d} S+ \\
\left(\int_{\partial_{g} \Omega^{e}}[\boldsymbol{N}]^{\mathrm{T}}\{\hat{\boldsymbol{F}}\}_{k} \mathrm{~d} S-\int_{\Omega^{e}}[\boldsymbol{B}]^{\mathrm{T}}[\boldsymbol{\sigma}]_{k} \mathrm{~d} V\right) .
\end{gathered}
$$

If $[\Delta \sigma]$ is taken as the linear term of the Taylor expansion of $[\sigma]$ at the time-discretized $k$ th iteration and the incremental constitutive relationships (15) and Equation (16) are adopted, the linearized form of Eq. (43) can be expressed as

$$
\left[\begin{array}{ll}
\boldsymbol{K}_{u u}^{\mathrm{e}} & \boldsymbol{K}_{u \lambda}^{\mathrm{e}}
\end{array}\right]\left\{\begin{array}{l}
\Delta \boldsymbol{u}_{\mathrm{h}}^{\mathrm{e}} \\
\Delta \lambda_{\mathrm{h}}^{\mathrm{e}}
\end{array}\right\}=\left\{\Delta \hat{\boldsymbol{F}}_{\mathrm{h}}^{\mathrm{e}}\right\}+\left\{\Delta \hat{\boldsymbol{F}}_{\mathrm{h}}^{\mathrm{e}}\right\}_{k}^{\mathrm{unb}}
$$

where

$$
\begin{aligned}
& \left\{\Delta \hat{F}_{\mathrm{h}}^{\mathrm{e}}\right\}=\int_{\partial_{g} \Omega^{e}}[\boldsymbol{N}]^{\mathrm{T}}\{\Delta \hat{\boldsymbol{F}}\} \mathrm{d} S, \\
& \left\{\Delta \hat{F}_{\mathrm{h}}^{\mathrm{e}}\right\}_{k}^{\mathrm{unb}}=\int_{\partial_{g} \Omega^{e}}[\boldsymbol{N}]^{\mathrm{T}}\{\hat{\boldsymbol{F}}\}_{k} \mathrm{~d} S- \\
& \int_{\Omega^{\mathrm{e}}}[\boldsymbol{B}]^{\mathrm{T}}[\boldsymbol{\sigma}]_{k} \mathrm{~d} V=\left\{\hat{F}_{\mathrm{h}}^{\mathrm{e}}\right\}_{k}-\int_{\Omega^{\mathrm{e}}}[\boldsymbol{B}]^{\mathrm{T}}[\boldsymbol{\sigma}]_{k} \mathrm{~d} V,
\end{aligned}
$$

The completed linearized Galerkin's equation becomes

$$
\left[\begin{array}{cc}
\boldsymbol{K}_{u u}^{\mathrm{e}} & \boldsymbol{K}_{u \lambda}^{\mathrm{e}} \\
\left(\boldsymbol{K}_{u \lambda}^{\mathrm{e}}\right)^{\mathrm{T}} & \boldsymbol{K}_{\lambda \lambda}
\end{array}\right]\left\{\begin{array}{c}
\Delta \boldsymbol{u}_{\mathrm{h}}^{\mathrm{e}} \\
\Delta \lambda_{\mathrm{h}}^{\mathrm{e}}
\end{array}\right\}=\left\{\begin{array}{c}
\Delta \hat{\boldsymbol{F}}_{\mathrm{h}}^{\mathrm{e}}+\left(\Delta \hat{\boldsymbol{F}}_{\mathrm{h}}^{\mathrm{e}}\right)_{k}^{\mathrm{unb}} \\
\mathbf{0}
\end{array}\right\} .
$$

Solutions of displacements and plastic multipliers at the $(k+1)$ th iteration can be obtained by

$$
\left\{\begin{array}{l}
\boldsymbol{u}_{\mathrm{h}}^{\mathrm{e}} \\
\lambda_{\mathrm{h}}^{\mathrm{e}}
\end{array}\right\}_{k+1}=\left\{\begin{array}{l}
\boldsymbol{u}_{\mathrm{h}}^{\mathrm{e}} \\
\lambda_{\mathrm{h}}^{\mathrm{e}}
\end{array}\right\}_{k}+\left\{\begin{array}{l}
\Delta \boldsymbol{u}_{\mathrm{h}}^{\mathrm{e}} \\
\Delta \lambda_{\mathrm{h}}^{\mathrm{e}}
\end{array}\right\} .
$$

It should be noted that the yield condition equation (38) is still not exactly satisfied at each iteration, but only the consistency condition is satisfied. However in the governing equation for the plastic flow, only linear terms of the Taylor expansion of the yield function are considered and the higher-order terms are truncated, which means that the Eq. (38) will not generally be satisfied at any stage of computation. Due to the coupled effect of $\boldsymbol{u}$ and $\boldsymbol{\lambda}$, we cannot use the same method as with $\left\{\boldsymbol{u}_{\mathrm{h}}^{\mathrm{e}}\right\}$ to treat $\left\{\lambda_{\mathrm{h}}^{\mathrm{e}}\right\}$. Instead, we need to design an alternative algorithm to adjust $\left\{\lambda_{h}^{e}\right\}$ to force $\lambda$ to satisfy 
the yield condition and then compute the unbalanced nodal forces to reflect this adjustment in the equilibrium equations. This algorithm will be described in the following section.

\subsection{The moving boundary technique and nonlocal elements}

Governing equations of the two coupled field, $\{\boldsymbol{u}\}$ and $\{\lambda\}$, consist of two equations: one is the equilibrium equation and the other the yield condition. These two sets of governing equations are associated with one another through flow rule and consistency condition. Therefore, there is no doubt that the linearized Galerkin's equation (47) is valid only for the domain where the material has entered the plastic stage and is under plastic loading. For the same domain, the yield condition is not involved in the governing equations in the following three cases: elastic loading and unloading, unloading from plastic stage, and reloading before reaching the new yield surface. In these three cases, the yield condition will not be satisfied and it will not enter the finite element formulation. On the other hand, for the same structures under loading, some parts of it may be in the elastic stage, whereas the other parts are experiencing plastic flow. This situation still results in the exclusion of the yield condition from the governing equations for some elements. In the present research, we use the moving boundary technique and nonlocal element to realize the exclusion and inclusion of yield condition in the Galerkin's equation by means of adjusting the degrees of freedom (DOFs) of the plastic multiplier field, $\{\lambda\}$.

Recall the definition of CRVE in Section 2.1. For the two dimensional cases, if domain $\Omega$ has unit thickness, the CRVE reduces to a square RVE with a side length of $l_{\mathrm{c}}$ (Fig. 3). For a discretized 2-D domain $\Omega$, a RVE is composed of all the elements inside a square region $A B C D$. All these elements inside this square region are called the nonlocal elements attached to the local element $e_{i}^{(0)}$, which is located in the center of the region. In other words, every finite element itself is not only a local element, but also has a set of adjacent finite elements attached to it. These adjacent are called nonlocal elements whose host element is the local element located in the center of the RVE. In Fig. 3, elements $e_{i}^{(j)}$, $j=0,1, \ldots, 8$, constitute a set of 2 -D nonlocal elements whose host element is a 2-D element $e_{i}^{(0)}$. Notice that a host element itself serves as one of its nonlocal elements.

According to the above definition, a local element is both a host element and a nonlocal element attached to some other host elements. Consequently the nonlocal elements for some host elements are overlapped within

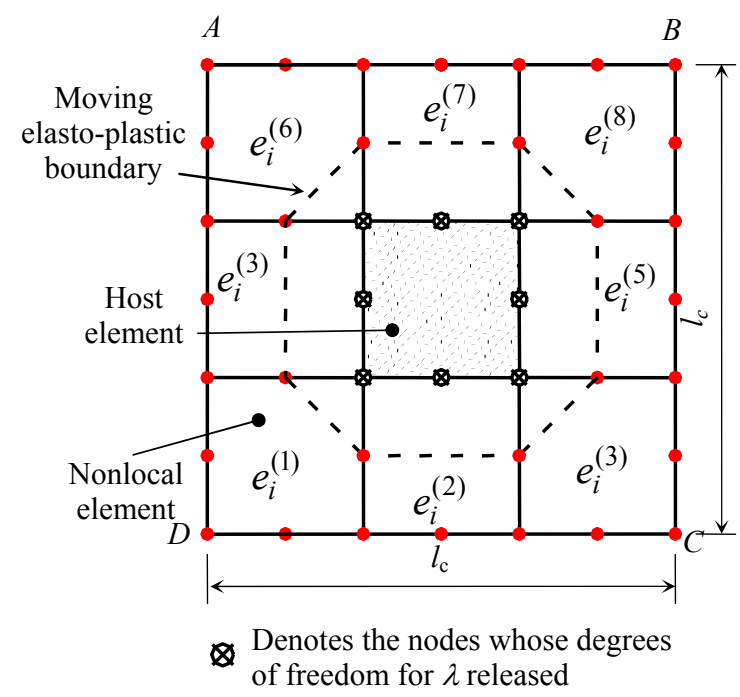

Fig. 3 The moving elasto-plastic boundary and nonlocal elements

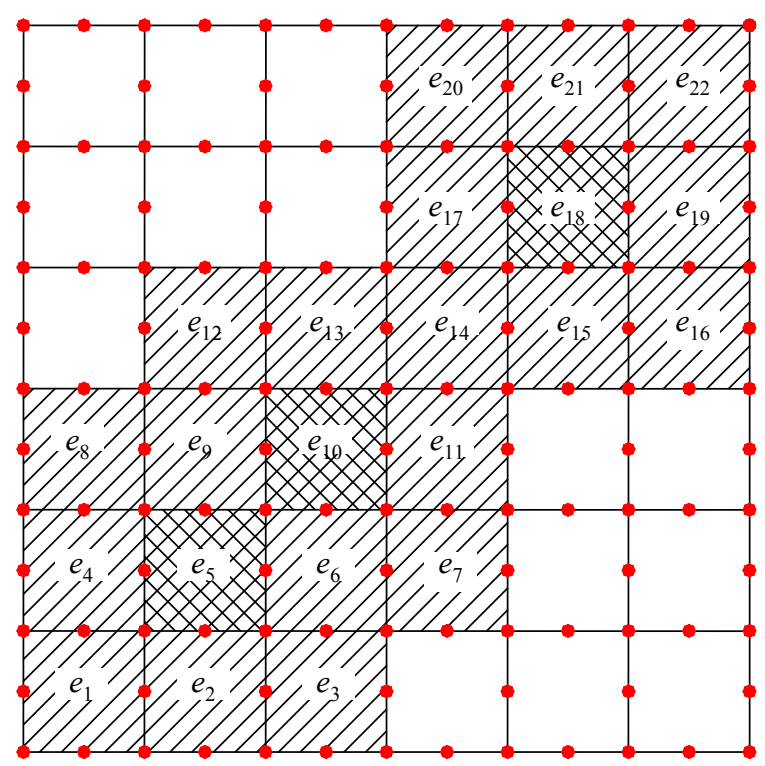

(a) Nonlocal finite-element discretization

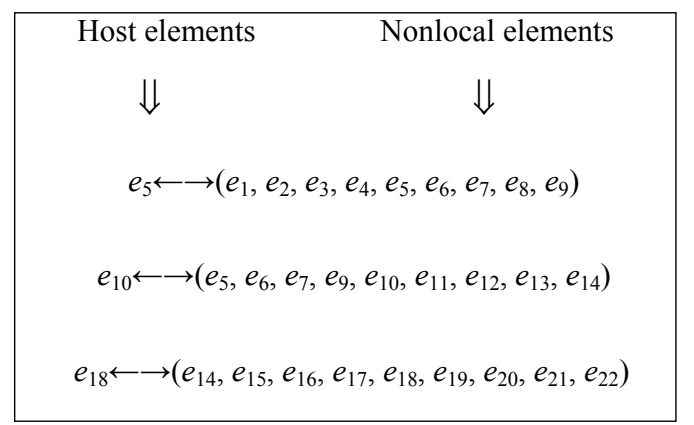

(b) Correspondence of host elements and nonlocal elements

Fig. 4. The overlapped nonlocal elements 
the discretized domain. For example, in Fig. 4(a), the nonlocal elements of the host elements $e_{5}, e_{10}$, and $e_{18}$ are overlapped (also see Fig. 4(b)).

The purpose of defining a set of nonlocal elements for a host element is to evaluate the nonlocal constitutive quantities of a local element. In the current study, the only nonlocal constitutive quantity is the equivalent plastic strain or plastic multiplier, $\lambda$. If at the $k$ th iteration of the $n$th load step, the stress state at some Gaussian integration point, say, $G P_{i}$, in the local element $e_{i}$ meets the yield condition, the whole element $e_{i}$ is thought as to be in yielding and all of the DOFs at its nodal points for $\lambda$, are activated, that is,

$$
\lambda_{h_{j}}^{e_{i}} \neq 0, j=1,2, \ldots, n_{e},
$$

where $\lambda_{h_{j}}^{e_{i}}$ represents the values of $\lambda$ at nodal points of element $e_{i}, h_{j}$ is the global node number, $n_{e}$ is the total number of nodal points in element $e_{i}$, and $j$ represents local node number, for example, $j=1,2, \ldots, 8$, for an 8 noded isoparametric element. In this way, the yield condition (39), enters the governing equations. On the

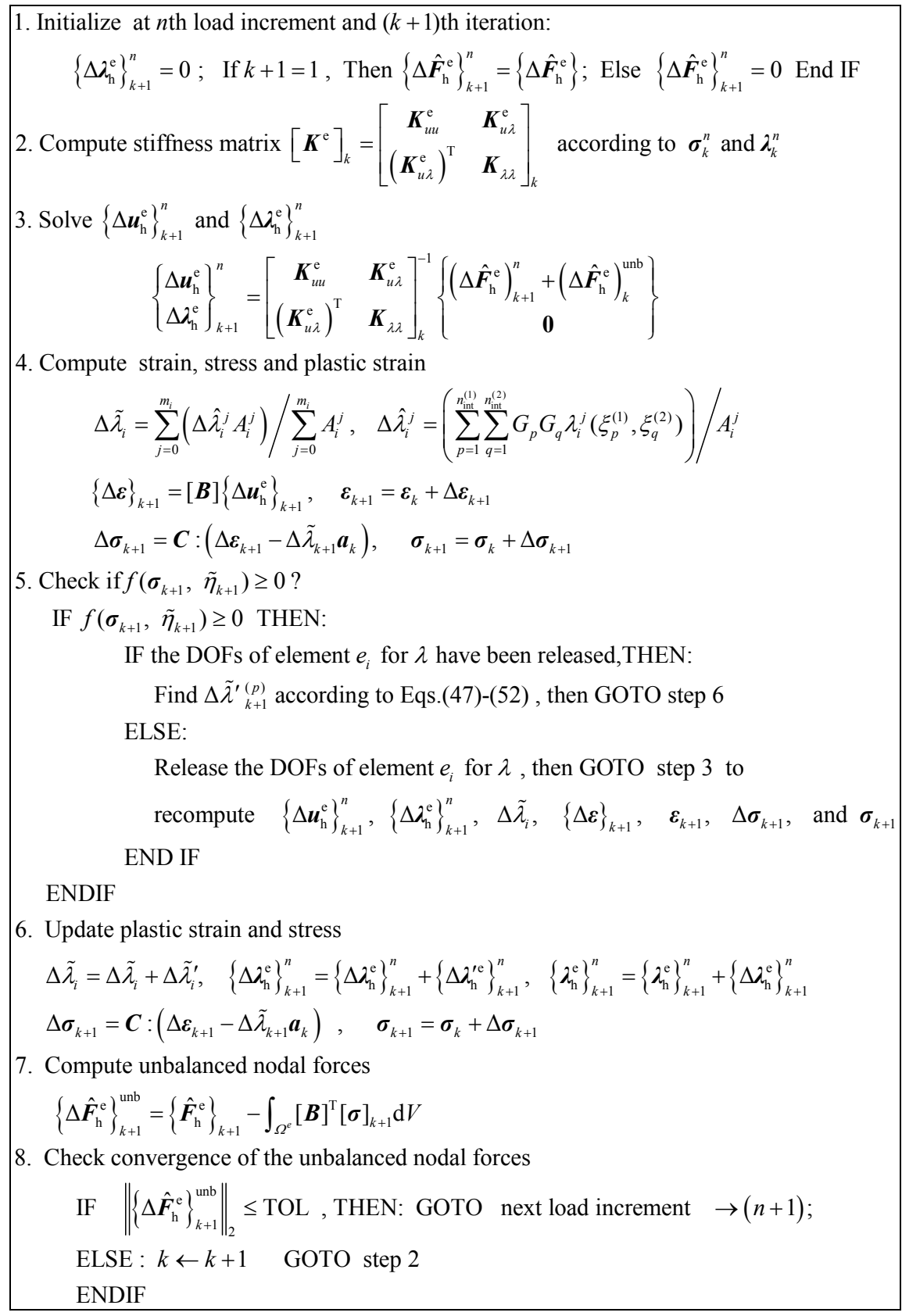

Box 1 The stress updating algorithm 
contrary, if any Gaussian integration points are in elastic or unloading stage from the yield surface or reloading, but not reaching the new yielding surface, the DOFs of its all nodal points for $\lambda$, will be inactive; that is,

$$
\lambda_{h_{j}}^{e_{i}}=0, j=1,2, \ldots, n_{e} .
$$

As a result, the yield condition will cease the governing equations. By making the DOFs of element nodal points for $\lambda$ active or inactive, the yield condition is controlled to come into or cease from playing in the governing equations. This approach is actually equivalent to moving the elasto-plastic boundary according to the stress state of local elements (Fig. 3).

If a uniform weighting function is assumed, the nonlocal plastic multiplier $\tilde{\lambda}_{i}$ for a host element $e_{i}$ can be evaluated by

$$
\tilde{\lambda}_{i}=\frac{\sum_{j=0}^{m_{i}}\left(\hat{\lambda}_{i}^{j} A_{i}^{(j)}\right)}{\sum_{j=0}^{m_{i}} A_{i}^{(j)}}
$$

where $\hat{\lambda}_{i}^{j}$ is the averaged $\lambda$ over the nonlocal element $e_{i}^{j}$ that is attached to host element $e_{i}$. In 2D cases, Gaussian numerical integration can be used to evaluate $\hat{\lambda}_{i}^{j}$, i.e.,

$$
\hat{\lambda}_{i}^{j}=\frac{\sum_{p=1}^{n_{\text {int }}^{(1)}} \sum_{q=1}^{n_{\text {int }}^{(2)}} G_{p} G_{q} \lambda_{i}^{j}\left(\xi_{p}^{(1)}, \xi_{q}^{(2)}\right)}{A_{i}^{j}},
$$

where $\xi_{p}^{(l)}, l=1,2$, is the coordinate of the $p$ th integration point in $l$ th dimension, and $G_{p}$ is the weight of the $p$ th integration point.

Because of $\mathrm{d} \lambda=\mathrm{d} \eta$ for the von Mises yield criterion, $\lambda=\eta$ and further $\tilde{\lambda}=\tilde{\eta}$ can be reasonably obtained. Provided that $\tilde{\lambda}_{i}$ is obtained for a local element $e_{i}$, we can substitute it into yield function (39) to check if the yield condition is satisfied. If it is, the unbalanced stresses will be computed. However for the higher-order finite element that involves nonlinear interpolation functions, stresses and strains are usually evaluated at Gaussian integration points. In this situation, $\tilde{\lambda}_{i}$ is taken to be constant over the local element $e_{i}$ and the values of $\lambda$ at all the Gaussian points of element $e_{i}$ are treated to be equal to $\tilde{\lambda}_{i}$. This treatment can be equivalently viewed as if the plastic strains are "smeared" over the whole local element. When a coarse mesh is used in the discretization, the approximate solution will be very rough. With the refinement of the mesh, the results will converge to the exact solution.

The significance of using nonlocal plastic strain instead of local plastic strain is that the variation of plastic strains in one element will affect the plastic strains in other elements that are within the characteristic region of a RVE. Due to the overlapping of the nonlocal elements and the application of the nonlocal plastic strains in the yield function, the nonlocality of plasticity is reflected in the interaction of plastic strains of local elements with one another.

The stress updating algorithm is summarized in Box 1.

\section{Numerical examples}

\subsection{Simulation of shear band formation in plane strain test}

Consider a specimen under plane strain test. The geometry and the loading conditions of the specimen are shown in Fig. 5. The specimen has the dimensions of $60 \mathrm{~mm} \times 120 \mathrm{~mm}$ and is placed on a level rigid plane. During the loading, the specimen's top face is constrained to remain horizontal and a given displacement is applied at the top edge. The deformation in the direction normal to the $X Y$ plane is kept to be zero. This is a

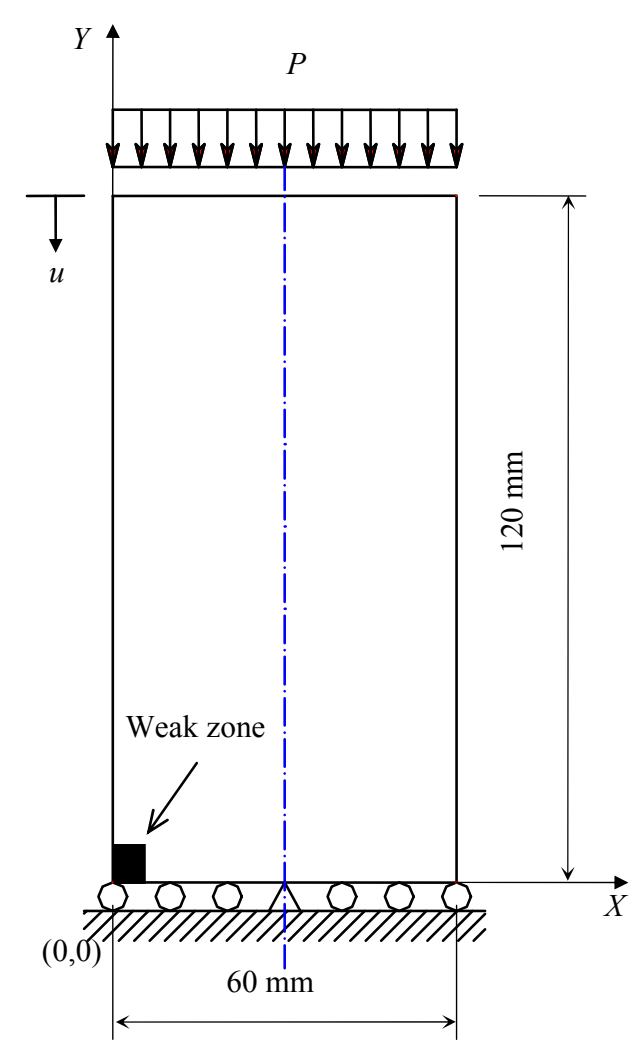

Fig. 5. Geometry and loading condition of the specimen under plane strain test 
classic plane strain problem that is used to illustrate the shear band formation in the strain softening stage of the responses [25]. The material parameters used in the computation is as follows: the elastic modulus $E_{0}=$ $11920 \mathrm{MPa}$ and the Poisson's ration $v=0.2$; the initial yield stress $\sigma_{\mathrm{Y}}=100 \mathrm{MPa}$ and the plastic modulus $E_{\mathrm{p}}=-0.1, E_{0}=-1192 \mathrm{MPa}$; and correspondingly, the tangential elasto-plastic modulus $E_{\mathrm{s}}^{\mathrm{ep}}=1322.22 \mathrm{MPa}$ (i.e., $\left.E_{\mathrm{s}}^{\mathrm{ep}}=\left(E_{0} E_{\mathrm{p}}\right) /\left(E_{0}+E_{\mathrm{p}}\right)\right)$. In the present study, the linear strain softening is assumed and the von Mises yield criterion is adopted through the computation in order to compare the results of the nonlocal plasticity model with that of the classical local theory. The characteristic length is taken to be $l_{\mathrm{c}}=14.7 \mathrm{~mm}$, which is equivalent to the internal length $c=3 \mathrm{~mm}$ that is adopted by de Borst and Pamin [25]. To trigger the onset of the shear band localization, a weak zone with $10 \%$ reduction of the initial yield strength is specified at the lower left corner of the specimen. Three different finite element meshes, 128-element (coarse), 512-element (medium), and 2 048-elements (fine), are used. Fig. 6 shows the mesh-dependent results when a classical local theory is used in the constitutive model. It can be seen
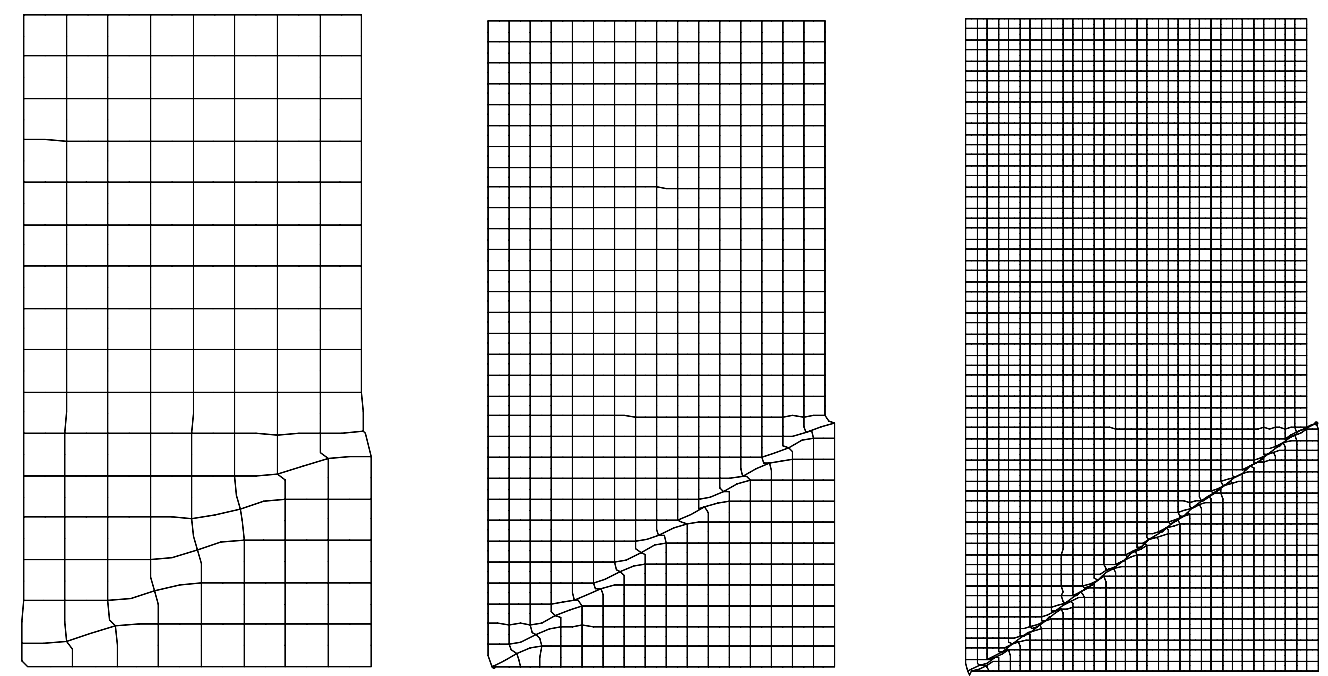

Fig. 6 Mesh-dependent deformation patterns for three different meshes
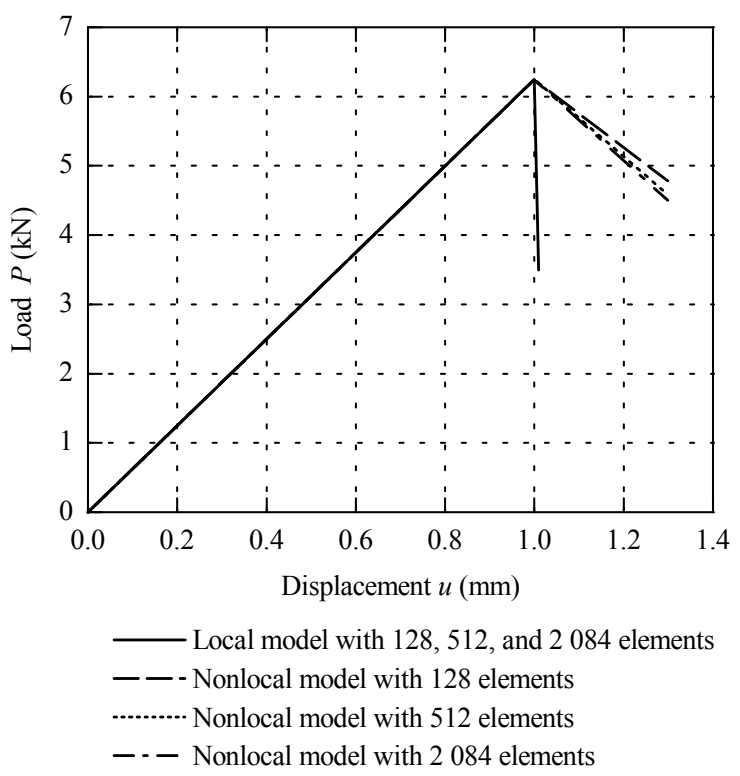

Fig. 7 Load-displacement curves for local and nonlocal models that the thickness of the shear band approaches to zero with mesh refinement. The load-displacement response shown in the Fig. 7 indicates the steepest-drop path after the peak load, which is typical of mesh-dependent response for the length ratio of the softening element to the elastic element $r_{s}$ greater than the tangential modulus ratio $r_{E}$ (see [36]).

Fig. 8 presents the deformation pattern for the nonlocal plasticity model proposed in this study. It can be observed that the deformation patterns for the three different meshes are similar and the thicknesses of the shear bands for the three meshes are approximately the same, which are nearly equal to the characteristic length of the RVE $l_{\mathrm{c}}=14.7 \mathrm{~mm}$. In Fig. 7, the load-displacement curve for the nonlocal plasticity model appears to converge to a stable descending branch. These results show that the proposed model can effectively lead to the mesh-independent simulation of the shear band localization, and the thickness of the shear band is an objective quantity, which is insensitive to the mesh refinement. 

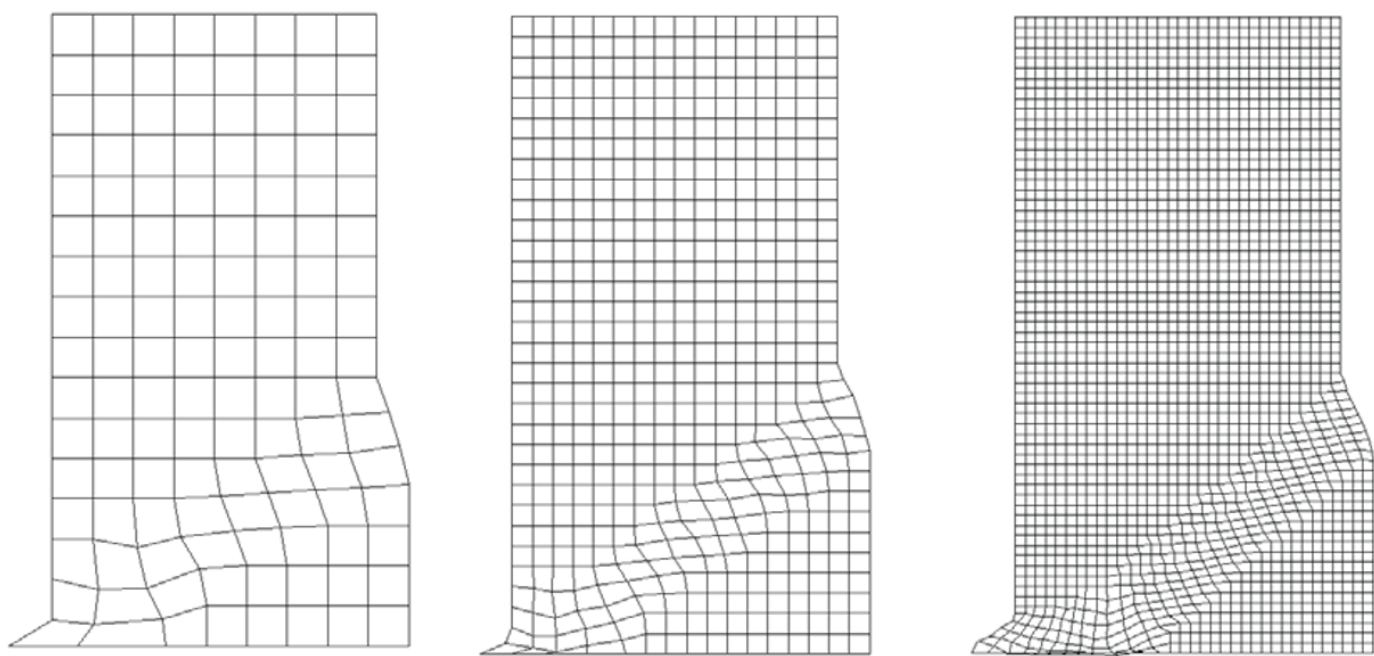

Fig. 8 Mesh-independent deformation patterns for three different meshes

\subsection{Simulation of borehole stability}

Micro-tunneling technique is often used to create boreholes for the installation of underground pipelines and sewerage. The stability of boreholes under railroads or highways is one of the problems that concern civil engineers. In this example, the proposed nonlocal plasticity model is used to analyze the stability of a borehole just below a highway. Fig. 9 shows the geometry, the position of the borehole, and the idealized mechanical model for the numerical analysis. For simplicity, the soil is assumed to be undrained in this case, so the von Mises model may be used in the analysis. The following material properties are used:

$$
\begin{aligned}
& E_{0}=11.2 \mathrm{MPa}, v=0.3, \\
& E_{\mathrm{p}}=-206.8 \mathrm{kPa}, \sigma_{\mathrm{Y}}=86 \mathrm{kPa} .
\end{aligned}
$$

To find the region of maximum stress, and in turn, obtain the localization pattern, no predefined weak zone is specified in the structure. The simulation result for the equivalent plastic strain is shown in Fig. 10. The maximum equivalent plastic strain is located at the horizontal semi-circle dividing points of the circumference of borehole. After the onset of the deformation localization, the shear band propagates slantingly

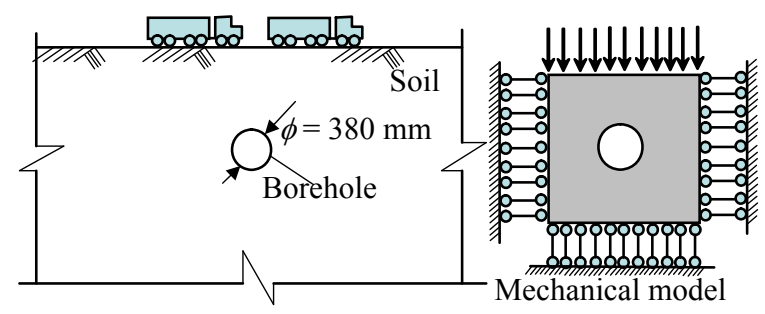

Fig. 9 Geometry and the position of the borehole

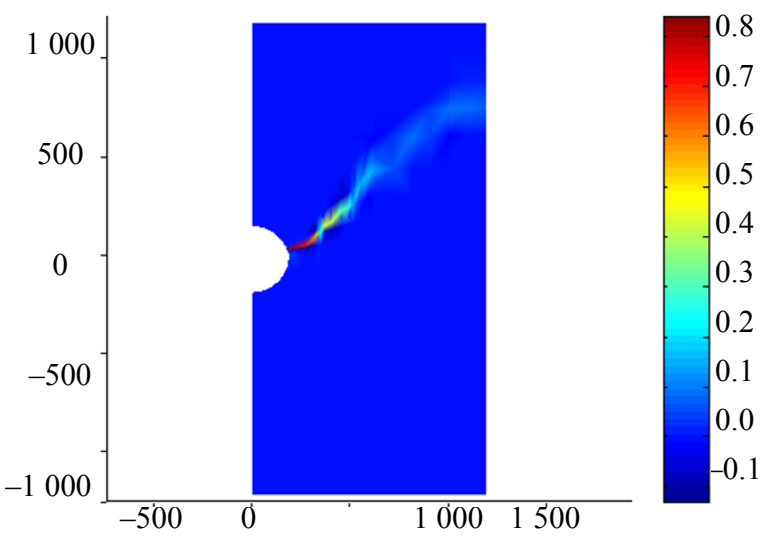

Fig. 10 Contour plot of the equivalent plastic strain in the soil around the borehole

upwards in an angle of about $40^{\circ}$ with respect to the horizontal axis. This result can help to improve the design of the underground pipeline below the ground traffic to avoid the unexpected failure of the pipeline, and also can help the engineers to judge the weak region of the soil around the borehole to avoid the borehole collapse during construction.

\section{Conclusions}

A new nonlocal plasticity model is proposed to simulate the shear band localization in geotechnical materials due to strain softening. In this model, the nonlocal internal state variable (taken to be the equivalent plastic strain in the current study) is introduced as a weighted averaging of its local counterpart over a representative volumetric element (RVE) of finite size. Applying the uniformly distributed weighting function and the truncated Taylor expansion of the local field, the character- 
istic length $l_{\mathrm{c}}$ of the RVE becomes equal to the width of the shear band, which is different from the other nonlocal models.

An algorithm is developed for the proposed model to solve the resulted nonlinear system of equations. In this algorithm, the nonlocal internal state variable is evaluated based on the integration of the local internal state variable over the RVE. Keeping the internal variable to be nonlocal and the other variables local, the differential consistency equation can be solved in the finite element context using only $C^{0}$ elements.

A solution technique, which is called the moving boundary technique with nonlocal elements, is developed to carry out the stress integration. The key points of this technique are:

(1) If the stress state of an element in an averaged sense satisfies the yield condition, the degrees of freedom for the plastic multiplier field at all nodal points of this element are activated. With load increased, more nodal points are activated if the plastic multipliers related to these nodal points are greater than zero. In this way, the elasto-plastic boundary moves with increased plastic deformation.

(2) Nonlocal plastic multiplier $\tilde{\lambda}$ for a local element is evaluated as an averaging of the local plastic multiplier over all of its nonlocal elements. The nonlocal elements for a host element (i.e. a local element), are included in a RVE. All the nonlocal elements for adjacent host elements are generally overlapping, and this overlapping property contributes substantially to the regularization of the mesh-dependent property of the classical local theory.

Two numerical examples, one for a plane strain compression test and another for the borehole stability, are conducted to demonstrate the mesh-independent property of the proposed model. The finite element analysis of the plane strain compression test shows that the proposed nonlocal model can yield mesh-independent simulation of the shear band localization. With the proposed model, the shear band pattern is insensitive to the mesh refinement, and the shear band width is approximately equal to the characteristic length $l_{\mathrm{c}}$ of the RVE. Application of the proposed model to the analysis of the borehole stability indicates the location of the initiation and propagation pattern of the shear band in the soils around the borehole during its collapse. The results can be helpful for engineers to find appropriate measures to prevent borehole failure.

\section{Acknowledgements}

The financial support for this work provided by Louisiana Board of Regents (RCS and Pfund) and high performance grid computing resources (HPC) provided by
LONI (Louisiana Optical Network Initiative) system are gratefully acknowledged.

\section{References}

[1] V. Tvergarrd, A. Needleman, K.K. Lo, Finite element analysis of localization in plasticity. In: J. T. Oden, G.F. Carey, eds., Finite Elements-Special problems in Solid Mechanics, Vol. 5 Prentice Hall, Englewood Cliffs, NJ, 1983: 94-157.

[2] M. Ortiz, Y. Leroy, A. Needleman, A finite element method for localized failure analysis, Computer Methods in Applied Mechanics and Engineering, 1987, 61(2): 189-214.

[3] H.E. Read, G.A. Hegemier, Strain softening of rock, soil and concrete-a review article, Mechanics and Materials, 1984, 27(3): 271-294.

[4] J.P. Bardet, A comprehensive review of strain localization in elastoplastic soils, Computers and Geotechnics, 1990, 10(3): 163-188.

[5] A. Needleman, Computational modeling of material failure, Applied Mechanics Review, 1994, 47(6): S34-S42.

[6] Y. Tomita, Simulation of plastic instabilities in solid mechanics, Applied Mechanics Reviews, 1994, 47(6): 171205.

[7] R. de Borst, Some recent issues in computational failure mechanics, International Journal for Numerical Methods in Engineering, 2001, 52(1-2): 63-95.

[8] R. de Borst, L.J. Sluys, H.B. Mühlhaus, et al., Fundamental issues in finite element analyses of localization of deformation, Engineering Computations, 1993, 10(2): 99-121.

[9] A. Needleman, M. Ortiz, Effect of boundaries and interfaces on shear-band localization, International Journal of Solids and Structures, 1991, 28(7): 859-877.

[10] R.I. Borja, R.A. Regueiro, Strain localization in frictional materials exhibiting displacement jumps, Computer Methods in Applied Mechanics and Engineering, 2001, 190(20-21): 2555-2580.

[11] D. Schaeffer, Instability and ill-posedness in the deformation of granular materials, International Journal of Numerical and Analytical Methods in Geomechanics, 1990, 14(4): 253-278.

[12] I. Vardoulakis, J. Sulem, Bifurcation Analysis in Geomechanics, London: Blackie Academic and Professional (Chapman \& Hall), 1995.

[13] R.A. Toupin, Elastic materials with couple-stresses, $A r$ chive for Rational Mechanics and Analysis, 1962, 11(1): 385-414.

[14] R.D. Mindlin, Micro-structure in linear elasticity, Archive for Rational Mechanics and Analysis, 1964, 16(1): 51-78.

[15] E.C. Aifantis, Gradient deformation models at nano, micro, and macro scales, Journal of Engineering Materials and Technology, 1999, 121(2): 189-202.

[16] E.C. Aifantis, On the role of gradients in the localization of deformation and fracture, International Journal of Engineering Science, 1992, 30(10): 1279-1299.

[17] H.B. Mühlhaus, E.C. Aifantis, A variational principle for 
gradient plasticity, International Journal of Solids and Structures, 1991, 28(7): 845-857.

[18] A.C. Eringen, Vistas of nonlocal continuum physics, International Journal of Engineering Science, 1992, 30(10): 1551-1565.

[19] A.C. Eringen, Theory of nonlocal plasticity, International Journal of Engineering Science, 1983, 21(7): 741751.

[20] Z.P. Bažant, F.B. Lin, Nonlocal smeared cracking model for concrete fracture, Journal of Structural Engineering, 1988, 114(11): 2493-2510.

[21] Z.P. Bažant, Imbricate continuum and its variational derivation, Journal of Engineering Mechanics, 1984, 110(12): 1693-1712.

[22] Z.P. Bažant, T.P. Chang, Nonlocal finite element analysis of strain softening solids, Journal of Engineering Mechanics, 1987, 113(1): 89-105.

[23] Z.P. Bažant, M. Jirásek, Nonlocal integral formulations of plasticity and damage: Survey and progress, Journal of Engineering Mechanics, 2002, 128(11): 1119-1149.

[24] R. de Borst, H.B. Mühlhaus, Gradient-dependent plasticity: Formulation and algorithm aspects, International Journal for Numerical Methods in Engineering, 1992, 35: 521-539.

[25] R. de Borst, J. Pamin, Some novel developments in finite element procedures for gradient-dependent plasticity, International Journal for Numerical Methods in Engineering, 1996, 39(14): 2477-2505.

[26] M. Jiŕasek, Z.P. Bažant, Inelastic Analysis of Structures, London and New York: Wiley \& Sons, 2002.

[27] R.H.J. Peerlings, M.G.D. Geers, R. de Borst, et al., A critical comparison of nonlocal and gradient-enhanced softening continua, International Journal of Solids and Structures, 2001, 38: 7723-7746.
[28] I. Vardoulakis, The 2nd gradient flow theory of plasticity, In: F. Darve, I. Vardoulakis, eds., Degradations and Instabilities in Geomaterials, Chapter 5, CISM, DIGAsponsored Course, Springer, 2004.

[29] J.C. Simo, T.J.R. Hughes, Computational Inelasticity, New York: Springer-Verlag, 1998.

[30] W. Han, B.D. Reddy, Plasticity: Mathematical Theory and Numerical Analysis, London: Springer, 1999.

[31] S. Ramaswamy, N. Aravas, Finite element implementation of gradient plasticity models Part I: Gradientdependent yield functions, Computer Methods in Applied Mechanics and Engineering, 1998, 163(1-4): 1132.

[32] S. Ramaswamy, N. Aravas, Finite element implementation of gradient plasticity models Part II: Gradientdependent evolution equations, Computer Methods in Applied Mechanics and Engineering, 1998, 163(1-4): 33-35.

[33] S. Wu, X. Wang, Comparison of boundary conditions of gradient elasticity and gradient plasticity, In: Inaugural International Conference of the Engineering Mechanics Institute, University of Minnesota, Minneapolis, Minnesota, 2008, May 18-21.

[34] W.C. Rheinboldt, Methods for Solving Systems of Nonlinear Equations, Philadelphia: Society for Industrial and Applied Mathematics, 1998.

[35] D.R.J. Owen, E. Hinton, Finite Elements in Plasticity: Theory and Practice, Swansea, U.K.: Pineridge Press Limited, 1980.

[36] S. Wu, X. Wang, Mesh Dependence and Nonlocal Regularization of One-Dimensional Strain Softening Plasticity, Journal of Engineering Mechanics, 2010, 136(11): 1354-1365.

(Editor: Dongju CHEN) 